\title{
Jugend, Sex und Sinus
}

\section{Die Entdeckung der Migrantenmilieus für die Sexualaufklärung Jugendlicher}

\author{
Thomas Kunz
}

Das Etikett »Migrationshintergrund « ist bei der Beschreibung von Lebenslagen nicht nur förderlich, wie eine Studie zur Sexualaufklärung junger Menschen zeigt.

Im letzten Jahr veröffentlichte die Bundeszentrale für gesundheitliche Aufklärung unter dem Titel »Sexualität und Migration: Milieuspezifische Zugangswege für die Sexualaufklärung Jugendlicher « neue Studienergebnisse zur Sexualaufklärung (vgl. BZgA 2010). Bezugspunkt der Untersuchung ist die Sinus-Milieustudie zu Menschen mit sogenanntem Migrationshintergrund, die auch in dieser Zeitschrift bereits Analysegegenstand war (vgl. Kunz 2008).

Neu und bemerkenswert ist an der Studie - im Vergleich zur vorhergehenden Sinusstudie - die Fokussierung auf die Altersgruppe der Jugendlichen. Im Mittelpunkt der nachfolgenden Überlegungen stehen Ergebnisse jener Studie in Bezug auf die Zusammensetzung der Milieus in der Gruppe der Jugendlichen mit Migrationshintergrund sowie daraus abgeleitete Erkenntnisse für milieuspezifisches Medienkonsumverhalten.

Um dem Anspruch, die Gruppe der Jugendlichen mit Migrationshintergrund zum Zwecke einer angemesseneren Sexualaufklärung stärker in den Blick zu nehmen, beauftragte die Bundeszentrale für gesundheitliche Aufklärung das Forschungsinstitut Sinus Sociovision mit der Durchführung einer repräsentativen Stichprobenuntersuchung. Die Bundes-

Prof. Dr. Thomas Kunz lehrt an der Fachhochschule Frankfurt am Main »Soziale Arbeit mit Kindern und Jugendlichen in einer Gesellschaft kultureller Vielfalt «. Bei dem Beitrag handelt es sich um die gekürzte Fassung eines Artikels aus den Zeitschriften "Migration und Soziale Arbeit « und »deutsche jugend «. E-Mail mtkunz@fb4.fh-frankfurt.de zentrale stieß in dieser Hinsicht kein neues Forschungsunterfangen an, vielmehr baut die Untersuchung auf der von der Bundeszentrale für gesundheitliche Aufklärung selbst im Jahr 2006 (mit-) initiierten Sinus-Migrantenmilieustudie auf. In Anbetracht des beachtlichen Anteils von Menschen mit Migrationshintergrund an der Altersgruppe der unter 25-Jährigen (ca. 27,2\%), der signifikant über dem allgemeinen Anteil von Personen mit Migrationshintergrund an der Gesamtbevölkerung liegt (ca. 18,6\%; vgl. Konsortium Bildungsberichterstattung 2006, $140 \mathrm{ff}$.), wird deutlich, dass es sich bei Jugendlichen und jungen Erwachsenen mit Migrationshintergrund demographisch um einen besonders relevanten Teil der Wohnbevölkerung Deutschlands handelt. Insofern scheint es aufgrund der demographischen Bedeutung nachvollziehbar und naheliegend, sich mit dieser Gruppe eingehend zu beschäftigen.

Dabei darf freilich nicht aus dem Blick geraten, dass eine Fremdzuschreibung, wie sie mittels des Etiketts »Migrationshintergrund « vorgenommen wird, nicht unproblematisch ist - was nicht nur in Bezug auf Jugendliche gilt (vgl. Hamburger, Stauf 2009). Die mit dieser Kategorie einhergehende homogenisierende Betrachtungsweise aggregiert unterschiedliche Einzelpersonen zu einem vermeintlich einheitlichen Kollektiv, deren einzig verbindendes Merkmal der ihnen zugeschriebene "Migrationshintergrund « ist. Die Frage liegt nahe, wie genau und mittels welcher Kriterien sich ein »deutscher Familienhintergrund " von einem »Migrationshintergrund « unterscheiden lässt? Oder anders gefragt: Bis zu welchem Zeitpunkt muss eine Migrationsgeschichte zurückreichen, um als solche zu gelten und die Attributierung zu rechtfertigen? Die Handhabung des Begriffes Migrationshintergrund lässt Spielräume und ist - entgegen dem Versprechen scheinbar klarer Begrifflichkeiten - ein weitaus schwierigeres Unterfangen, als es mit Verweis auf amtliche Definitionen anmutet.
Das primäre Ziel der Studie der Bundeszentrale für gesundheitliche Aufklärung war es, neue Zugangswege für die Sexualaufklärung Jugendlicher mit Migrationshintergrund $\mathrm{zu}$ erschließen (vgl. BZgA 2010, 5). Für die Vertiefung mit Blick auf Jugendliche wurden im Rahmen der Studie 608 Personen mit Migrationshintergrund im Alter von 14 bis 17 Jahren befragt (289 Mädchen, 319 Jungen). Knapp über die Hälfte von ihnen (53\%) ist in Deutschland geboren. Der Rest von ihnen (47\%) hat eigene Migrationserfahrungen (vgl. BZgA 2010, 13).

\section{Sinus-Milieuansatz}

In Anlehnung an den Sinus-Milieuansatz und dessen Dimension "soziale Lage« (unterschieden in »niedrig «, »mittel «, »hoch«) sowie die Grundorientierungen (»Tradition «, »Modernisierung «, »Neuidentifikation «) wurden für die Gruppe der Menschen mit Migrationshintergrund in Deutschland acht unterschiedliche Milieus identifiziert, die untereinander Überschneidungen aufweisen: ein religiös-verwurzeltes Milieu, ein traditionelles (Gast-) Arbeitermilieu, ein statusorientiertes Milieu, ein entwurzeltes Milieu, ein intellektuell-kosmopolitisches Milieu, ein adaptives bürgerliches Milieu, ein multikulturelles Performermilieu sowie ein hedonistisch-subkulturelles Milieu (vgl. vhw 2009).

Aus dieser Zuordnung zur Milieulandschaft wurden Aussagen über die Migranten-Population in Deutschland abgeleitet. Diese erweise sich demnach als facettenreich, Negativ-Klischees über Einwanderer würden durch die Studie zudem widerlegt. Insbesondere der Einfluss religiöser Traditionen werde oft überschätzt. Quer durch die Migrantenmilieus werde zudem eine mangelnde Integrationsbereitschaft seitens der Aufnahmegesellschaft beklagt.

Die vorfindbaren Migrantenmilieus unterscheiden sich, so eine weitere Er- 
kenntnis, weniger nach ethnischer Herkunft und sozialer Lage als viel mehr nach ihren Wertvorstellungen, Lebensstilen und ästhetischen Vorlieben. Es bestünden Milieu-Bildungen und MilieuÜberschneidungen über unterschiedliche Herkunftskulturen hinweg. Milieubedingte Bindekräfte seien stärker als herkunftsspezifische (vgl. Kunz 2008).

\section{Ergebnisse}

Spannend erscheinen die Befunde der Untersuchung der Bundeszentrale für gesundheitliche Aufklärung in Bezug auf Jugendliche und die Lebensphase Jugend, wenn man insbesondere auf Unterschiede (oder gegebenenfalls Ähnlichkeiten) im Vergleich zur bisherigen Untersuchung der Migrantenmilieus achtet, die keine altersspezifische Differenzierung vornahm. Hier lassen sich mehrere bemerkenswerte Ergebnisse identifizieren, die geeignet sind, dramatisierende Befunde anderer Studien zu konterkarieren. So beispielsweise die Rolle und Bedeutung der Glaubens- und Religionszugehörigkeit bei Jugendlichen mit Migrationshintergrund, wie sie beispielsweise zuletzt in einer Studie des Kriminologischen Forschungsinstitutes Niedersachsen beobachtbar war (vgl. Baier, Pfeiffer, Rabold 2010). Dort wurde behauptet: »Eine hohe christliche Religiosität senkt die Gewaltbereitschaft, eine hohe islamische Religiosität erhöht sie indirekt, in dem sie Faktoren verstärkt, die die Gewaltbereitschaft fördern. Zudem zeigt sich, dass eine hohe Religiosität die Integration von jugendlichen Migranten nicht behindert; dies gilt allerdings erneut nicht für muslimische Migranten. " (Baier, Pfeiffer, Rabold 2010: 9) Das Zitat und noch mehr die öffentliche Rezeption dieser Studie belegten, mit welch großer Bereitschaft, Hinweise auf Religiosität und insbesondere muslimische Religionszugehörigkeit herangezogen werden, um im Integrationsdiskurs vermeintlich begründet über Defizite und Probleme, die den MigrantInnen und einer bestimmten Glaubensrichtung ursächlich zuzurechnen seien, klagen zu können.

Wurde die Bedeutung des religiös-verwurzelten Milieus bereits mit Blick auf die Gesamtbetrachtung der Sinus-MigrantenMilieus in Deutschland relativiert, so bekräftigt die Studie in Bezug auf Jugendliche diese Relativierung nochmals: Wurde der
Anteil des religiös-verwurzelten Milieus schon in der Gesamtstudie lediglich auf 7 Prozent beziffert, betrage dieser Milieuanteil in der Gruppe der Jugendlichen im Alter von 14 bis 17 Jahren nur 3 Prozent (vgl. BZgA 2010, 17). Obgleich darauf hingewiesen wird, dass immerhin 83 Prozent der Jugendlichen mit Migrationshintergrund einer Religions- und Glaubensgemeinschaft angehören (vgl. ebd., 13), ist der Sachverhalt somit geeignet, als Indiz für eine medial vermittelte Überbetonung muslimischer Religionszugehörigkeit im Migrationsdiskurs gedeutet zu werden, die sich in einer problemfixierten und dramatisierenden Berichterstattung niederschlägt.

Ein weiterer Aspekt, der mit dem geringeren Anteil an Milieus mit traditioneller Grundorientierung einhergeht, ist der vergleichsweise höhere Anteil Jugendlicher an Milieus mit einer an Modernisierung ausgerichteten Grundorientierung. Jugendliche mit Migrationshintergrund verfügen demnach im Vergleich überproportional über eine an den Grundhaltungen »Modernisierung « und "Neuidentifikation « angelehnte Orientierung (vgl. BZgA 2010, 13).

Die Tendenz zu modernen Lebensauffassungen und Lebensweisen ist gleichwohl keine Gewähr für konfliktfreie Lebensperspektiven und Einstellungen der Jugendlichen: »Im Hedonistisch-subkulturellen Milieu (33\%) distanziert man sich (teilweise aggressiv) von der Mehrheitsgesellschaft. Dies äußert sich in der Unangepasstheit im Outfit, im Verhalten und in der Sprache. Konflikte mit Autoritäten (Eltern, Lehrer, Vorgesetzte, Polizei) sind dabei vorprogrammiert. " (BZgA 2010, 15)

Ähnlich problematisch sei es im sogenannten Entwurzelten Milieu, welchem 12 Prozent der Jugendlichen mit Migrationshintergrund zuzuordnen seien. Hier »leben die Jugendlichen zwischen Welten und Kulturen. Sie haben Orientierungsprobleme in der Aufnahmegesellschaft; ihre häufig prekären Lebenssituationen und die Ausbildungsdefizite führen zu Gefühlen von Deklassierung und Marginalisierung. «(BZgA 2010, 15)

Beide Zitate können als Hinweis dafür gelten, wie in der Studie der Bundeszentrale für gesundheitliche Aufklärung wenn auch vereinzelt - versucht wird, mit ethnisch-spezifischen Erklärungsmustern Milieuspezifika zu erklären. Statt dessen wäre es hinsichtlich der angesprochenen
Problemlagen - beispielsweise bei »Konflikten mit Autoritäten « - vielleicht ergiebiger zu fragen, inwieweit nicht bereits der Hinweis auf die Lebensphase Jugend, auf entsprechende Pubertäts- und Adoleszenzprozesse ausreichend Erklärungsangebote unterbreitet, um die angesprochenen Verhaltensweisen als einfach jugendphasentypisch erklärlich zu machen.

An anderer Stelle wiederum konstatiert die Studie, dass beispielsweise das »Freizeitverhalten der jugendlichen Migrantinnen und Migranten [...] sehr jugendtypisch « (BZgA 2010, 7) sei. So banal dieser Befund anmuten mag, so ist er doch wichtig in Hinsicht auf die Gefahr einer segregierenden Bildung von Milieuräumen oder einer Sonderbetrachtungsweise von Jugendlichen mit Migrationshintergrund. Nicht zuletzt beim Thema des Mediennutzungsverhaltens lassen sich beispielsweise Hinweise auf Gemeinsamkeiten finden: "Wie bei Jugendlichen in Deutschland insgesamt, sind auch bei Jugendlichen mit Migrationshintergrund die Zeitschriften Bravo und Bravo Girl am weitesten verbreitet, unabhängig von Milieu und Herkunftskultur." (BZgA 2010, 40)

Die Befunde der Studie, so kann abschließend festhalten werden, sind trotz aller Ambivalenz in Bezug auf den Migrantenmilieuansatz also geeignet, in der Integrationsdebatte geläufige Dramatisierungen zu entkräften, wenn resümierend festgehalten wird: "Insgesamt zeigt sich sowohl hinsichtlich spezifischer Themenbereiche (wie z. B. Sexualität, Liebe, Partnerschaft) wie auch auf übergeordneter Einstellungs- und Verhaltensebene, dass Milieuunterschiede - und damit die jeweils real gelebten Alltagswelten - deutlich prägender sind als die Zugehörigkeit zu einer bestimmten Migrantenpopulation oder zur deutschen Bevölkerung. « (BZgA 2010, 8)

Eine Differenzierung der Ansprachewege scheint - folgt man der Bundeszentrale für gesundheitliche Aufklärung - dennoch geboten: »Nicht alle jugendlichen Adressaten können mit dem gleichen $\mathrm{Me}$ dium und vor allem nicht mit dem Medium >Print $<$ erreicht werden. Für die unterschiedlichen Milieus müssen unterschiedliche Medien, Aufmachungen und Inhalte bereitgestellt werden, um den unterschiedlichen Lebenswelten und Informationsbedürfnissen gerecht zu werden.«(BZgA 2010, 8). 


\section{Bewertung}

Die Übertragung des Milieu-Ansatzes auf die Thematik der Sexualaufklärung von Jugendlichen provoziert dennoch eine grundsätzliche Kritik, die in etwas veränderter Weise bereits dem altersgruppenunspezifischen Sinus-Migrantenmilieuansatz entgegenzuhalten war: Es stellt sich zum Ersten die Frage, wieso beim Thema Sexualaufklärung Jugendlicher das Thema Migration bislang nicht in einer Weise einbezogen und berücksichtigt wurde, die seiner demographischen Bedeutung angemessen gewesen wäre? Zum Zweiten ist zu fragen, wieso spezifische, also separate Migranten-Milieuräume erhoben werden? Besteht hierdurch nicht die Gefahr, gerade diejenigen Jugendlichen mit und ohne Migrationshintergrund voneinander unterscheidbar $\mathrm{zu}$ machen, deren Milieus und Lebenswelten sich hinsichtlich ihrer sozialen Lage und Grundorientierungen gar nicht (mehr) unterscheiden (lassen)?

Gerade der Aspekt der sozialen Lage rechtfertigt die Annahme, dass nicht der Migrationshintergrund das Milieu prägt, als vielmehr die sozioökonomische Lage. Nicht spezifische ethnische Gruppenzugehörigkeiten, angenommene kulturelle Differenz begründen Milieuunterschiede, als vielmehr der Umstand, dass Menschen mit Migrationshintergrund in bestimmten sozioökonomischen (vorwiegend schwierigen) Lagen überrepräsentiert sind - was sich nachteilig auf Bildungs- und Berufschancen sowie generell negativ auf deren gesellschaftliche Teilhabechancen auswirkt.

Die Frage liegt deshalb nahe, ob, sofern der Migrationshintergrund so markant hervorgehoben wird, letztlich nicht milieutechnisch separiert wird, was in weiten Teilen längst schon zusammengewachsen ist? Diesen Einwand stützt die Studie der Bundeszentrale für gesundheitliche Aufklärung letztlich selbst, wenn dort festgehalten wird: »Es stellte sich heraus, dass Menschen des gleichen Milieus, aber mit unterschiedlichem Migrationshintergrund, mehr miteinander verbindet als mit dem Rest ihrer Landsleute aus anderen Milieus. «(BZgA 2010, 10)

Mit Blick auf die zunehmende Bedeutung der Kategorie Migrationshintergrund ist folglich für eine Fachdebatte zu plädieren, die nach der grundsätzlich separierenden Dynamik der Begriffsver- wendung fragt und die Diskussion um die inhaltliche und zeitliche Reichweite der Definition des Migrationshintergrundes in den Mittelpunkt stellt - und insbesondere die Grenzen des Erklärungsgehaltes auslotet. Während auch Verfechter des Sinus-Milieuansatzes, der sich auf die Gesamtbevölkerung bezieht, ganz explizit ein » integriertes Milieumodell « favorisieren, also einen Ansatz, der die milieubezogene Separation zwischen Mehrheitsgesellschaft und Migranten zu überwinden versucht (vgl. vhw 2009, 8, 42 ff.), scheint die Studie der Bundeszentrale für gesundheitliche Aufklärung diese Einsicht (zumindest noch) nicht zu teilen.

\section{Fazit}

Nach der Lektüre der neuen Studie der Bundeszentrale für gesundheitliche Aufklärung über Jugendliche mit Migrationshintergrund stellt man erneut fest: Es sollte endlich über die Ausgestaltung dessen nachgedacht werden, was an dieser Stelle mit dem Begriff der »interkulturellen Öff- nung der Bevölkerungsstatistik « nur plakativ umrissen werden kann.

Diese Notwendigkeit besteht nicht zuletzt deshalb, weil die Zuweisung des Etiketts »Migrationshintergrund « im Sinne einer Fremdzuschreibung Integrationsprozesse durchaus zu konterkarieren vermag. Damit ist der Umstand gemeint, dass eine gewünschte Identifizierung mit der Mehrheitsgesellschaft durch die Zuschreibung "Migrationshintergrund « erschwert, wenn nicht gar unterlaufen werden kann. Man ist im besten Falle eben bloß »Deutscher mit Migrationshintergrund «.

Diese Zumutung macht die Jugendphase von Menschen mit Migrationshintergrund gewiss nicht einfacher. Identitätsbildungen, die diese Zuschreibung in Gestalt von Bekenntnissen zu - oder gar Überbetonungen von - Herkunftsnationalitäten in jeweilige Lebensentwürfe einzubauen versuchen, werden dann zu einem späteren Zeitpunkt wiederum gerne illustrierend herangezogen, um in der Einwanderungsgesellschaft über vermeintliche Integrationsmisserfolge von Menschen mit Migrationshintergrund zu räsonieren.

\section{Literatur}

Baier, Dirk/Pfeiffer, Christian/Rabold, Susann 2010: Kinder und Jugendliche in Deutschland. Gewalterfahrungen, Integration, Medienkonsum. Zweiter Bericht zum ge-meinsamen Forschungsprojekt des Bundesministeriums des Innern und des KFN, KFN-Forschungsbericht Nr. 109, Hannover.

Beauftragte der Bundesregierung für Migration, Flüchtlinge und Integration (Hg.) 2010: 8. Bericht der Beauftragten der Bundesregierung für Migration, Flüchtlinge und Integration über die Lage der Ausländerinnen und Ausländer in Deutschland, Berlin. Bundeszentrale für gesundheitliche Aufklärung (BZgA) (Hg.) 2010: Sexualität und Migrati-on: Milieuspezifische Zugangswege für die Sexualaufklärung Jugendlicher. Ergebnisse einer repräsentativen Untersuchung der Lebenswelten von 14- bis 17-jährigen Jugendlichen mit Migrationshintergrund, Köln.

Hamburger, Franz/ Stauf, Eva 2009: »Migrationshintergrund « zwischen Statistik und Stigma, in: Diehm, Isabell/Gomolla, Mechtild et al. (Hg:) 2009: Schüler. Wissen für Lehrer. Migration, Seelze, 30-31.

Konsortium Bildungsberichterstattung (Hg.) 2006: Bildung in Deutschland. Ein indikatorengestützter Bericht mit einer Analyse zu Bildung und Migration, Bielefeld.

Kunz, Thomas 2008: Gibt es Parallelmilieus in Deutschland?, in: Blätter der Wohlfahrtspflege, Heft 4/2008, S. 129-132.

Kunz, Thomas 2010: When Ayse goes Pop. Das Medium Jugendzeitschrift als Ort stereotyper genderspezifischer Fremdheits- und Eigenbilder, in: Baros, Wasilios/Hamburger, Franz/Mecheril, Paul (Hg.) 2010: Festschrift für Georg Auernheimer. Zwischen Praxis, Politik und Wissenschaft. Die vielfältigen Referenzen interkultureller Bildung, Berlin, 155-169.

Sachverständigenrat deutscher Stiftungen für Integration und Migration 2010: Einwanderungsgesellschaft 2010. Jahresgutachten 2010 mit Integrationsbarometer, Berlin. vhw - Bundesverband Wohnen und Stadtentwicklung e.V. (Hg.) 2009: MigrantenMilieus. Ein Kompass für die Stadtgesellschaft, Berlin. 